\title{
Optical Properties of Nanostructured Silver Embedded by Electro-Thermo-Diffusion in Opal Photonic Crystal
}

\author{
V. L. Veisman ${ }^{1}$, S. G. Romanov' ${ }^{2}$ V. G. Solovyev ${ }^{1}$, M. V. Yanikov ${ }^{1}$ \\ ${ }^{1}$ Department of Physics, Faculty of Physics and Mathematics, Pskov State University, \\ Lenin Square 2, 180000 Pskov, Russia; \\ ${ }^{2}$ Institute of Optics, Information and Photonics, University of Erlangen-Nuremberg, \\ Haber Str. 9a, 91058 Erlangen, Germany; \\ Ioffe Physical Technical Institute, \\ Polytechnicheskaya Street, 26, 194021 St. Petersburg, Russia
}

\begin{abstract}
Novel nanocomposite materials $\mathrm{Ag}$ / opal have been prepared by electro-thermo-diffusion of silver in opal template. Optical properties of these photonic crystals have been studied by angle-resolved reflectance spectroscopy. Interpretation of the observed optical spectra has been made on the basis of the Bragg diffraction and the Fano resonance between diffracted in $\mathrm{Ag} / \mathrm{opal}$ composite photonic crystal electromagnetic waves and those resonantly scattered by silver dendrites.
\end{abstract}

Keywords: Fano resonance, nanostructured silver, opal photonic crystals.

\section{INTRODUCTION}

Architectures combining conductive network and photonic crystal functionality pave a way for novel generation of efficient solar cells and lighting devices. To meet this demand we prepared the respective nanocomposite $\mathrm{Ag} / \mathrm{opal}$ by electro-thermo-diffusion of silver in opal template.

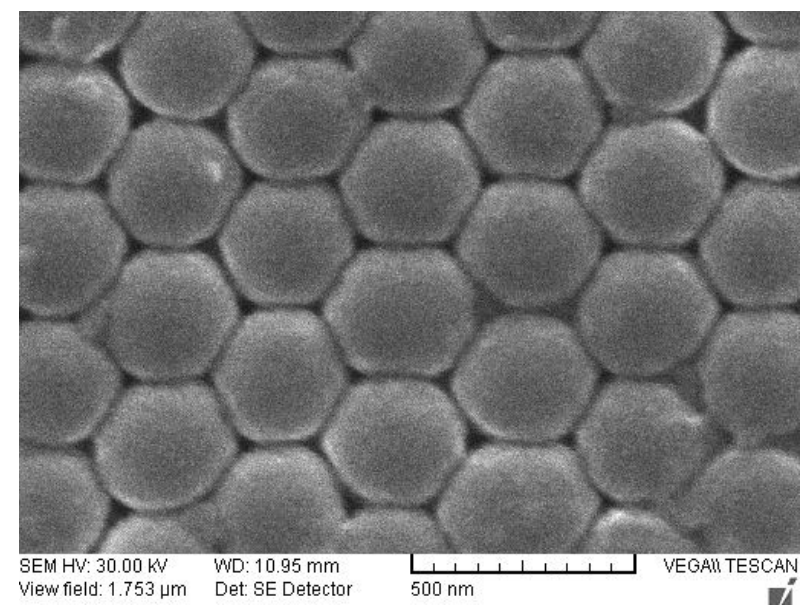

Fig. 1. SEM image of the opal matrix under study.

Self-assembled synthetic opals consist of silica or polymer beads that are assembled in closely packed face centered cubic (FCC) lattice [1] (Fig. 1). Since the diameters $D$ of beads are in the range of hundreds nanometers, opals and opal-based metal-dielectric composites are widely used as 3-dimensional photonic [2] and hybrid plasmonic-photonic crystals [3] for the visible light.

\section{MATERIALS AND METHODS}

Silica opal samples were characterized by «VEGA // LMU Tescan» Scanning Electronic Microscope in the Daugavpils University. According to SEM images (Fig. 1), the mean sphere diameter of the bare opal under study is $D \approx 288 \mathrm{~nm}$.

The electro-thermo-diffusion of silver in the opal matrix $\left(10 \times 10 \times 2 \mathrm{~mm}^{3}\right.$ slab $)$ has been carried out under electric field of $E=1.7 \mathrm{kV} / \mathrm{cm}$ at temperature of $\mathrm{T}=(664 \pm 2) \mathrm{K}$. The electrolysis process lasted for 2.5 hours. During this time the electric current was increased from $2.6 \mu \mathrm{A}$ up to the saturation value of $1.3 \mathrm{~mA}$. Therefore one can estimate the silver ion concentration after electrolysis as $\sim 10^{20} \mathrm{~cm}^{-3}$.

Angle-resolved reflectance spectra of the parent opal matrix as well as those of the opal-based nanocomposite $\mathrm{Ag} / \mathrm{opal}$ have been measured under illumination by white light from a tungsten lamp. Reflection spectra have been acquired by USB650 Red Tide spectrometer (Ocean Optics).

ISSN 1691-5402 


\section{RESULTS AND DISCUSSION}

Normalized to the maximum value reflectance spectra of opal template and those of nanocomposite $\mathrm{Ag} / \mathrm{opal}$ at two different angles of light incidence are shown in Fig. 2.

Reflectance spectra of all the samples demonstrate the zero order diffraction resonance that depends on the angle of light incidence $\theta$. The shift of the central wavelength $\lambda$ of the Bragg resonance (Fig. 3) obeys the combined Bragg $2 a \cos \beta=\lambda / n_{\text {eff }}$ and Snell's ( $n_{\text {eff }} \sin \beta=\sin \theta$ ) laws:

$$
\lambda^{2}=4 a^{2} n_{e f f}^{2}-4 a^{2} \sin ^{2} \theta,
$$

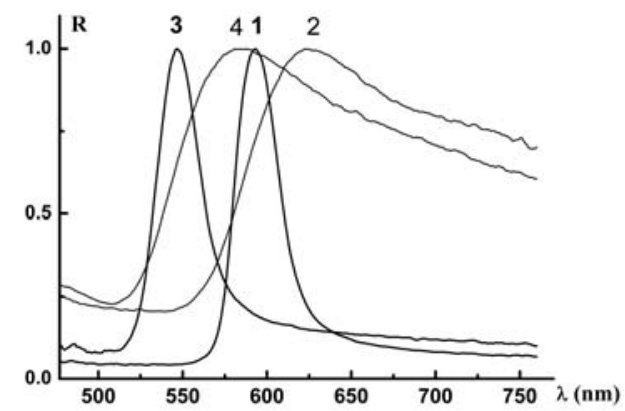

Fig. 2. Normalized reflectance spectra of opal template $(1,3)$ and those of nanocomposite $\mathrm{Ag} / \mathrm{opal}(2,4)$ at the angles of incidence

$$
15^{\circ}(1,2) \text { and } 35^{\circ}(3,4) \text {. }
$$

where $a=0.816 D$ is the interplane distance for (111) planes of the FCC lattice and $n_{\text {eff }}$ represents the effective refractive index of the photonic crystal.

The reflectance maxima in the Ag / opal nanocomposite spectra are shifted to the longer wavelengths as compared with those in the spectra of parent opal matrix, because the effective refractive index of composite ( $n_{\text {composite }} \approx 1.44$ ) is higher than that of the opal template ( $n_{\text {opal }} \approx 1.33$ ).

The striking observation is the pronounced distortion of diffraction resonance band in the reflectance spectra of $\mathrm{Ag} / \mathrm{opal}$ composite (Fig. 2, curves 2,4) that contrasts to quite symmetric Bragg resonance shape in the spectra of bare opal (Fig. 2, curves 1,3 ). To our opinion, this phenomenon is the manifestation of the Fano resonance [4] - [7] between diffracted in $\mathrm{Ag} /$ opal composite photonic crystal electromagnetic waves and those resonantly scattered by silver dendrites.

Under this assumption, the resonance profile could be approximated by Fano formula

$$
R=\frac{(\varepsilon+q)^{2}}{\varepsilon^{2}+1}, \text { where } \varepsilon=\frac{D}{\lambda}
$$

is the reduced energy (wavenumber or frequency), $q$ represents the phenomenological parameter describing the relative strength of the Bragg resonance and the

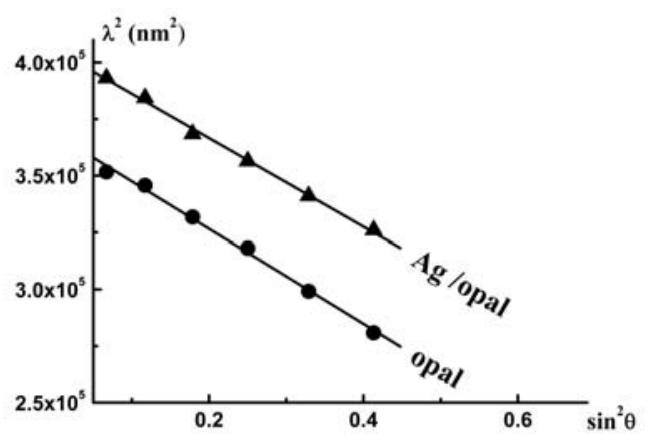

Fig. 3. Angular dispersions of the (111) Bragg resonances in the opal template and in the $\mathrm{Ag} /$ opal photonic crystal.

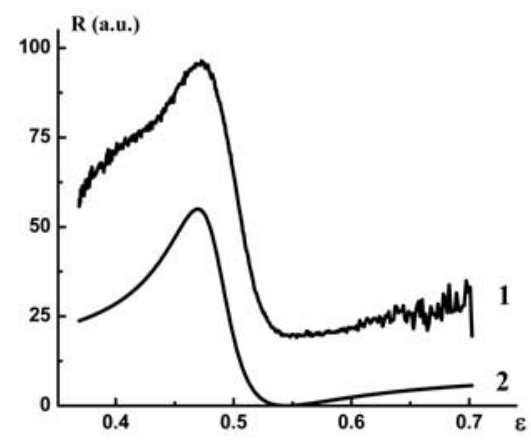

Fig. 4. Reflectance spectrum of composite $\mathrm{Ag} / \mathrm{opal}$ at the angle of incidence $15^{\circ}$ and its approximation with Fano formula $(q=-2)$.

broadband dendrite plasmon scattering.

Fig. 4 demonstrates rather good agreement between the Fano theory and our experimental results.

\section{CONCLUSIONS}

The light diffraction in $\mathrm{Ag} /$ opal photonic crystals prepared by electro-thermo-diffusion of silver in opal matrix is affected by the light scattering at silver dendrites. This interaction leads to Fano-type distortion of the Bragg resonance shape.

\section{$\mathrm{V}$ ACKNOWLEDGEMENTS}

The authors wish to thank M. I. Samoilovich for providing high-quality bulk opal matrices, V. I. Gerbreder and A. S. Ogurcov for having taken the SEM images, G. S. Cema and A. A. Gonyan for their assistance in some experiments, A. I. Vanin and S. I. Grashchenkov for fruitful discussions.

This work was supported by the Ministry of Education and Science of Russian Federation under the project 2.4128.2011 "Complex Investigation of 
Physical Properties of Nanocomposites Based on Regular Porous Matrices".

\section{REFERENCES}

[1] V. G. Balakirev, V. N. Bogomolov, V. V. Zhuravlev Y. A. Kumzerov, V. P. Petranovskii, S. G. Romanov, L. A. Samoilovich, "Three-dimensional superlattices in opals", Crystallography Reports, vol. 38, pp. 348-353, 1993.

[2] K. Busch, S. Lölkes, R. B. Wehrspohn, H. Föll, Eds., Photonic crystals: Advances in design, fabrication, and characterization. Weinheim: Wiley-VCH, 2004.

[3] S. G. Romanov, A. V. Korovin, A. Regensburger, U. Peschel, "Hybrid colloidal plasmonic-photonic crystals", Advanced Materials, vol. 23, pp.2515-2533,2011.
[4] U. Fano, "Effects of configuration interaction on intensities and phase shifts", Phys. Rev., vol. 124, pp. 1866-1878, 1961.

[5] A. E. Miroshnichenko, S. Flach, Yu. S. Kivshar, "Fano resonances in nanoscale structures", Reviews of Modern Physics, vol. 82, pp. 2257-2298, 2010.

[6] M. Yanikov, V. Veisman, A. Gonyan et al., "Experimental investigation of physical properties of nanostructured silver, embedded by electro-thermo-diffusion into opal porous matrix", Vestnik PskovSU. Series "Natural Sciences, Physics and Mathematics", issue 5, pp. 196-201, Pskov, 2014.

[7] M. Yanikov, V. Veisman, S. G. Romanov, V. Solovyev, "Experimental study of Fano resonance in university course of physics", The Problems of Educational Physical Experiment, $25^{\text {th }}$ Collection of Articles, pp. 96-98, Moscow, 2015. 